\title{
Elaboração e caracterização de doce cremoso de caqui com adição de sementes da araucária
}

\author{
Preparation and characterization of a sweet creamy persimmon pudding with the \\ addition of Brazilian pine seeds
}

\author{
Aline Balbinotti Bolzan ${ }^{1 *}$, Edimir Andrade Pereira1 \\ 1 Universidade Tecnológica Federal do Paraná (UTFPR), Departamento de Química, Campus Pato Branco, Pato Branco/PR - Brasil
}

${ }^{*}$ Corresponding Author:

Aline Balbinotti Bolzan, Universidade Tecnológica Federal do Paraná (UTFPR), Departamento de Química, Campus Pato Branco, Via do conhecimento, km 1, CEP: 85503-390, Pato Branco/PR - Brasil, e-mail: alinebbalbinotti@hotmail.com

Cite as: Preparation and characterization of a sweet creamy persimmon pudding with the addition of Brazilian pine seeds. Braz. J. Food Technol., v. 20, e2016061, 2017.

Received: May 23, 2016; Accepted: May 23, 2017

\section{Resumo}

A inovação é um dos pilares para o alcance do desenvolvimento sustentável e, para a inserção de um novo produto alimentício no mercado, abordagens que englobem pesquisas em ciência e tecnologia se fazem necessárias. Visando propor uma alternativa para o uso do pinhão e o aproveitamento de frutos de clima temperado, cultivados no Estado do Paraná, foi elaborado um doce cremoso de caqui, substituindo-se parcialmente a polpa por diferentes concentrações de pinhão nas formulações (0, 5 e 10\%). Foram realizadas análises de sólidos solúveis, atividade de água, pH, acidez total titulável, teor de umidade, cinzas, cor e textura, além da caracterização microbiológica dos produtos. Atendendo aos padrões de identidade e qualidade quanto às características físicas, químicas e microbiológicas, a inserção de sementes de pinhão e a consequente redução no teor de polpa promoveram, do ponto de vista tecnológico, o desenvolvimento adequado deste produto inovador. Os valores da atividade de água, a concentração de açúcares e a baixa acidez observados no produto tornaram o ambiente desfavorável ao desenvolvimento de micro-organismos, condições estas associadas às boas práticas de fabricação. A alteração na formulação padrão influenciou na análise instrumental do perfil de textura, devido à presença de amido da semente de pinhão. Os parâmetros de cor apresentaram resultados de baixa luminosidade, o que reporta a produtos pouco translúcidos, o que é característico de alimentos de alto teor de açúcares, concentrados por evaporação. No espaço de cor notou-se a expressão da cromaticidade $a^{*} e b^{*}$, que apontam para a predominância das cores amarelo $e$ vermelho, característicos do fruto de origem. As investigações abordadas revelam a aplicabilidade tecnológica do pinhão, contribuindo para a agregação de valor através da diversificação de produtos e mostrando-se como uma alternativa para geração de recursos financeiros para pequenos produtores rurais através do extrativismo, garantindo a conservação de espécies da Floresta com Araucária.

Palavras-chave: Diospyrus kaki L.; Araucaria angustifolia; Processamento; Caracterização físico-química e microbiológica; Análise instrumental de textura e cor.

\section{Abstract}

Innovation is one of the pillars for achieving sustainable development, and in order to insert a new food product onto the market one must use approaches that encompass scientific and technological research. With a view to proposing an alternative use for Brazilian pine seeds (Araucaria angustifolia), and temperate climate fruits cultivated in the State of Paraná, Brazil, a creamy persimmon pudding was elaborated with partial substitution of the fruit by different concentrations of Brazilian pine seeds $(0,5$ and $10 \%)$. The following properties of the products were analysed: soluble solids, water activity, $\mathrm{pH}$, total acidity, moisture, ash, colour, texture and the microbiological characteristics. Complying with the standards of identity and quality and also with the physical, chemical and microbiological characteristics, from the technological point of view, the insertion of pine seeds and consequent reduction in pulp content allowed for the adequate development of this innovative product. The values for water activity, the sugar content and the low acidity level observed in the sweet creamy pudding made the 
Elaboração e caracterização de doce cremoso de caqui com adição de sementes da araucária

Bolzan, A. B.; Pereira, E. A.

ambience unfavourable for microbial development, associated with good manufacturing practices. Due to the presence of starch in the Brazilian pine seeds, the change in the standard formulation influenced the instrumental texture profile analysis. The colour parameters showed low luminosity, indicating only slightly translucent products, characteristic of high-sugar content foods concentrated by evaporation, and the expression of the $a^{*}$ and $b^{*}$ chromaticity indicated the predominance of the colours yellow and red, characteristic of the original fruit. The investigations carried out in this study revealed the technological applicability of the Brazilian pine. This contribute to the adding value via product diversification, as an alternative to generate financial resources for small farmers by way of extractivism, ensuring the conservation of forest species such as Araucaria.

Keywords: Diospyrus kaki L.; Araucaria angustifolia; Processing; Physicochemical and microbiological characterization; Texture and colour analyses.

\section{Introdução}

A Floresta Ombrófila Mista caracteriza-se pela emergência das copas da Araucaria angustifolia (Bertol.) O. Kuntze, sendo esta a única espécie arbórea deste gênero que ocorre naturalmente no Brasil (CITADINI-ZANETTE et al., 2011). Nas últimas décadas, houve intensa exploração da espécie, colocando-a, em 1992, na lista oficial de espécies da flora brasileira ameaçadas de extinção do Instituto Brasileiro do Meio Ambiente e dos Recursos Naturais Renováveis (IBAMA). Em 2001, uma resolução do Conselho Nacional do Meio Ambiente (CONAMA) (BRASIL, 2001b) suspendeu o corte e a exploração. A forma mais segura de garantir sua preservação é a exploração econômica de seus produtos não madeiráveis, dentre os quais o pinhão (FIGUEIREDO FILHO et al., 2011). Através da Portaria $n^{\circ} 048$, o Instituto Ambiental do Paraná (PARANÁ, 2011), no Art. $3 .^{\circ}$, foi fixada a data de 15 de abril para início da colheita, do transporte e da comercialização do pinhão. A cadeia extrativa do pinhão é bastante simplificada, não sendo verificado nenhum processo industrial, diferentemente do que acontece com outras amêndoas. Esse é um dos maiores obstáculos à comercialização, tendo em vista as reduzidas técnicas de conservação (CONAB, 2014).

A semente da Araucária, o pinhão, é nutritiva e muito apreciada. A produção anual varia de 40 até 200 pinhas por planta, dependendo da idade da Araucária (FIGUEIREDO FILHO et al., 2011). Segundo Schveitzer et al. (2014), independentemente do modo de consumo, seja cru, cozido ou assado, o pinhão é rico em minerais, como fósforo, zinco e, principalmente, ferro, além de ser excelente fonte de lipídeos e proteínas.

O caqui é uma das principais frutas do outono e sua boa colocação no mercado é devida à pequena diversidade de frutas disponíveis nesse período (CAMARGO FILHO et al., 2003). Sua maior produção está nos Estados de São Paulo, Rio Grande do Sul, Paraná e Minas Gerais, pelas condições climáticas (MARTINELI et al., 2013). Em 2014, a produção foi de 182,3 mil toneladas, segundo dados apresentados pela Secretaria de Estado de
Agricultura, Pecuária e Abastecimento de Minas Gerais (MINAS GERAIS, 2015).

A atração pela cultura do caquizeiro é explicada pela qualidade nutricional do fruto, constituindo uma boa fonte de fibras, carboidratos, vitaminas, polifenóis e sais minerais. Um dos principais atrativos do caqui é sua capacidade antioxidante, através do ácido ascórbico (ELIAS et al., 2008). Além disso, apresenta grande quantidade de polpa, excelente sabor, aparência e cor atraentes, garantindo boa aceitação do fruto no mercado (NUNES et al., 2009).

Mesmo não existindo um padrão de identidade de doces, por se tratarem de produtos tradicionais, certas características ou definições são comumente aceitas (VENDRUSCOLO et al., 2009) e utilizadas neste trabalho. Doce em pasta é o produto resultante do processamento adequado das partes comestíveis de vegetais com açúcares, pectina e ajustador de $\mathrm{pH}$, até uma consistência apropriada. Entendem-se como "vegetais", todas as frutas, os tubérculos e outras partes comestíveis reconhecidamente apropriadas para elaboração de doces. O produto pode ser classificado como doce cremoso quando a pasta for homogênea e de consistência mole, não devendo oferecer resistência nem possibilidade de corte (BRASIL, 1978). A Resolução da ANVISA RDC n 272/2005 passa a utilizar o termo genérico "produtos de frutas", incluindo geleias, compotas de frutas, etc., que devem ser designados por denominações consagradas pelo uso (BRASIL, 2005). Esses alimentos contêm geralmente uma grande quantidade de açúcar, o que inibe o crescimento de microrganismos (SOUZA, 2001); mesmo assim, doces necessitam de um controle de qualidade para garantir a segurança alimentar (AKUTSU et al., 2005).

A determinação instrumental da cor, na indústria de alimentos, fornece informações importantes sobre a qualidade, a aceitação e a vida-de-prateleira dos produtos. Em derivados de frutas, como a geleia, este atributo é importante e pode ser percebido sensorialmente; contudo, o teste hedônico tem aplicação limitada nessa avaliação. Portanto, gera-se a necessidade do emprego 
Elaboração e caracterização de doce cremoso de caqui com adição de sementes da araucária

Bolzan, A. B.; Pereira, E. A.

de técnicas analíticas para eliminar erros das variações do julgamento visual subjetivo. A escala CIELAB é atualmente o sistema mais utilizado na descrição quantitativa da cor (NEIRO et al., 2013). Assim como a cor dos alimentos, a textura é outro atributo-chave na avaliação da qualidade e da aceitabilidade de um produto, desde a decisão sobre a prontidão para a colheita até a operação de processamento e a vida-de-prateleira. A avaliação deste atributo vai além do descritivo sensorial através de análise instrumental (CHEN; OPARA, 2013), avaliando o perfil de textura (TPA)

O desenvolvimento de produtos com ingredientes típicos atende não apenas a consumidores regionais, pois, quando sua introdução em alimentos convencionais propicia uma combinação harmônica, há uma diversificação na oferta de produtos industrializados, os quais, além de preservarem a riqueza de sabores, atingem paladares exigentes e qualificados. A possibilidade de comercialização do doce de caqui com pinhão foi verificada mediante testes sensoriais de aceitação e intenção de compra por Bolzan e Pereira (2016).

O estudo visa processar e caracterizar o doce cremoso de caqui com adição de sementes da araucária.

\section{Material e métodos}

\subsection{Matéria-prima e métodos de produção}

Os caquis da variedade Fuyu em estádio ótimo de comercialização - maduros, cor da casca amarelo avermelhada, textura firme e ausência de injúrias ou doenças - foram adquiridos no comércio da cidade de Pato Branco, Paraná. Em seguida, foram encaminhados para o Laboratório, onde se procedeu a lavagem em água corrente e sanitização em solução clorada a 100 ppm, durante $15 \mathrm{~min}$. Os frutos foram cortados, retirando-se os caroços, a casca e demais partes indesejáveis ao processo, e então triturados em um liquidificador industrial por 3 min, até obtenção da polpa, com posterior refino através de peneira de metal com malha de $0,8 \mathrm{~mm}$. Os pinhões foram adquiridos na cidade de Mangueirinha-Paraná e foram submetidos ao cozimento em panela de pressão durante $45 \mathrm{~min}$. Em seguida, as sementes foram descascadas e trituradas em liquidificador industrial por $5 \mathrm{~min}$. As formulações utilizadas no estudo (Tabela 1) foram baseadas em testes preliminares descritos por Bolzan e Pereira (2016).

Os doces foram preparados em "tacho abertos" (pressão atmosférica), com agitação contínua e temperatura de ebulição de $103^{\circ} \mathrm{C}$. Para produção da amostra controle, a polpa de caqui foi aquecida e, em seguida, o açúcar foi gradativamente adicionado (1:1). Para as formulações B e C, parte da polpa de caqui foi substituída por pinhão. O caqui apresenta quantidades expressivas de pectina (SENTER et al., 1991) e, por se tratar de um doce cremoso, não se fez necessária a adição deste ingrediente. Neste processo, foi monitorada a concentração de sólidos solúveis, a qual, de acordo com a legislação, não deve ser inferior a $55^{\circ}$ Brix (BRASIL, 1978). Próximo ao ponto final de cozimento, foi adicionado $1 \%$ de ácido cítrico, para abaixamento da acidez, evitando-se, assim, a hidrólise da pectina. O tempo de cocção foi semelhante para todas as formulações. Após esta etapa, envasou-se o doce a quente $\left(85^{\circ} \mathrm{C}\right)$ em embalagens de vidro, previamente esterilizadas, e fechadas com tampa de metal. O resfriamento foi feito em tanque por meio da circulação de água fria. Os produtos foram armazenados à temperatura ambiente média de 17,0 $\pm 3,5^{\circ} \mathrm{C}($ IAPAR, 2015).

O processamento e as análises dos doces foram realizados nos Laboratórios de Tecnologia de Alimentos, Fruticultura, e no Laboratório de Qualidade Agroindustrial (LAQUA), da Universidade Tecnológica Federal do Paraná (UTFPR) campus Pato Branco, no mês de maio de 2014. Todas as análises foram realizadas em triplicata.

\subsection{Análise microbiológica}

As determinações seguiram a Resolução CNNPA n 12 , de 2 de janeiro de 2001 (BRASIL, 2001a). Os parâmetros analisados foram: contagem de Unidade Formadora de Colônia de Bolores e Leveduras, Estafilococos Coagulase Positiva, Salmonella sp., Número Mais Provável de coliformes totais a $35^{\circ} \mathrm{C}$ e coliformes termotolerantes a $45^{\circ} \mathrm{C}$.

\subsection{Parâmetros físico-químicos}

A atividade água $\left(A_{w}\right)$ foi determinada através do aparelho Novasina MasterLab AW, com a temperatura da câmara de medição ajustada em $25^{\circ} \mathrm{C}$ e mantida constante até estabilização. O teor de sólidos solúveis (SS) foi medido por refratometria, utilizando-se um Refratômetro Portátil (45 a $82^{\circ}$ Brix) Itref-82. Medições do potencial de hidrogeniônico foram realizadas usando pHmetro microprocessador Digital DEL LAB, modelo DLA $\mathrm{pH}$. As análises de umidade, teor de cinzas, acidez total titulável (ATT) foram realizadas de acordo com o Instituto Adolfo Lutz (IAL, 2008).

Tabela 1. Formulações dos doces mistos de caqui cv Fuyu adicionados de pinhão.

\begin{tabular}{cccc|} 
Ingredientes & \multicolumn{3}{c|}{ Amostras } \\
\cline { 2 - 4 }$(\%)$ & $\mathbf{A}$ & $\mathbf{B}$ & $\mathbf{C}$ \\
\hline Caqui & 50,00 & 45,00 & 40,00 \\
Pinhão & 00,00 & 5,00 & 10,00 \\
Açúcar & 50,00 & 50,00 & 50,00 \\
\hline
\end{tabular}

A: doce de caqui sem pinhão; B: doce de caqui com $5 \%$ de pinhão; C: doce de caqui com $10 \%$ de pinhão. 
Elaboração e caracterização de doce cremoso de caqui com adição de sementes da araucária Bolzan, A. B.; Pereira, E. A.

\subsection{Análise de cor}

A análise da cor foi avaliada por refletância no espaço de cor CIELab, usando colorímetro Konica Minolta CR-400, com iluminante D65, em que se apontam determinados parâmetros de cor luminosidade $\left(L^{*}\right)$ e as coordenadas $a^{*} e b^{*}$, bem como os valores do ângulo de tonalidade (hue, $h^{\star}$ ), a pureza da cor (chroma ou saturação, $C^{\star}$ ) e o cálculo diferença total da cor da amostra em relação ao padrão, conforme a Equação 1:

$$
\Delta \mathrm{E}^{*}=\left(\Delta \mathrm{L}^{2}+\Delta \mathrm{a}^{2}+\Delta \mathrm{b}^{2}\right)^{1 / 2}
$$

\subsection{Análise de textura}

O perfil de textura (TPA) foi analisado utilizando-se o texturômetro Stable Micro Systems Modelo TA - XT2i (Goldaming, England) com probe cilíndrico (P/0.5R). Os parâmetros de medição foram os seguintes: velocidade do pré-teste: 5,0 s; Velocidade de teste: 2,0 s; Velocidade de pós-teste: 2,0 s, e distância: $10 \mathrm{~mm}$. Foram avaliados os parâmetros de dureza, adesividade, elasticidade, coesividade e gomosidade dos doces.

\subsection{Análise estatística}

Os resultados foram expressos em médias e desvio padrão, sendo submetidos à análise de variância (ANOVA) e ao teste de comparação de Tukey $(p<0,05)$. Foram realizadas análises estatísticas multivariadas: análise de agrupamento, análise de fator e análise de componentes principais $(A C P)$, realizadas pelo software Statistica ${ }^{\circledR} 8.0$, com dados previamente normalizados por meio da função Standardize.

\section{Resultados e discussão}

\subsection{Análise microbiológica}

Na Tabela 2, são apresentados os resultados das análises microbiológicas dos doces cremosos de caqui cv Fuyu, com e sem adição de sementes da araucária. Os doces devem obedecer ao padrão preconizado pela Resolução CNNPA n 12, de 2 de janeiro de 2001, a qual preconiza que bolores e leveduras não devem exceder $10^{4}$ por grama em produtos de frutas (BRASIL, 2001a).
Note-se que bolores e leveduras não excederam $10^{4}$ por grama nos doces analisados; dessa forma, os resultados apresentaram conformidade com os níveis exigidos pela legislação. Nenhuma das amostras apresentou contaminação por estafilococos coagulase positiva, Salmonella sp., nem por coliformes a 35 e $45^{\circ} \mathrm{C}$. Portanto, os produtos estavam aptos para o consumo, o que decorreu de boas práticas no processamento do produto desenvolvido, como sanificação adequada das frutas e dos equipamentos utilizados, além da efetividade dos métodos de conservação empregados.

Doces de mamão, abóbora, figo, laranja, goiaba e jabuticaba foram analisados por Kato et al. (2013) e nenhum produto apresentou contaminação de mesófilos aeróbios, coliformes totais, coliformes termotolerantes e bolores e leveduras. Peçanha et al. (2006) analisaram goiabadas do tipo cascão, cujos resultados indicaram ausência de coliformes totais e fecais em todas as amostras. As contagens de mesófilos aeróbios variaram entre 10 e $10^{2}$ UFC/g e de fungos filamentosos e leveduras situaram-se entre 10 e $10^{3} \mathrm{UFC} / \mathrm{g}$.

\subsection{Parâmetros físico-químicos}

Na Tabela 3, são apresentados os valores obtidos nas análises físico-químicas dos doces cremosos de caqui cv Fuyu com e sem adição de sementes da araucária. Não foram observadas diferenças estatísticas entre as amostras, devido à substituição de até $10 \%$ da polpa por farinha de pinhão. Determinou-se o ponto final de cozimento do doce em 70,39 $\pm 0,70{ }^{\circ}$ Brix, apresentando consistência cremosa. Segundo Miquelim (2006), este teor de sólidos solúveis garante a conservação de produtos de frutas em temperatura ambiente. Os resultados estão próximos aos encontrados por Leite Júnior et al. (2013), em doce de manga acrescido de okara, variando de 61,4 a 70,8 ${ }^{\circ} \mathrm{Brix}$, durante o armazenamento, e similares aos de Querido et al. (2013), na formulação de geleia de caqui com $60{ }^{\circ}$ Brix de polpa da fruta, e aos de Caetano et al. (2012), que analisaram geleia de acerola com 66,92 a 67,97 ${ }^{\circ}$ Brix. Os sólidos solúveis estão relacionados diretamente com a formação do gel, pois as moléculas de água interferem na estrutura da rede tridimensional. Além disso, o açúcar age como um conservante, reprimindo o crescimento microbiano, pelo fato de aumentar a pressão osmótica com

Tabela 2. Resultados das análises microbiológicas dos doces de caqui cv Fuyu adicionados de pinhão.

\begin{tabular}{lccc}
\multicolumn{1}{c}{ Parâmetros } & \multicolumn{1}{c}{ Amostra } \\
\cline { 2 - 4 } & A & B & C \\
Bolores e leveduras $\left(\mathrm{g} \mathrm{mL}^{-1}\right)$ & $2 \times 10^{1}$ & - & $5 \times 10^{1}$ \\
Estafilococos Coagulase positiva & - & - & - \\
Salmonella sp. & - & $<0,3$ & - \\
Coliformes totais a $35^{\circ} \mathrm{C}\left(\mathrm{g} \mathrm{mL}^{-1}\right)$ & $<0,3$ & $<0,3$ \\
Coliformes termotolerantes a $45^{\circ} \mathrm{C}\left(\mathrm{g} \mathrm{mL}^{-1}\right)$ & $<0,3<$ & $<0,3$ \\
\hline
\end{tabular}

A: doce de caqui sem pinhão; B: doce de caqui com $5 \%$ de pinhão; C: doce de caqui com $10 \%$ de pinhão. 
Elaboração e caracterização de doce cremoso de caqui com adição de sementes da araucária

Bolzan, A. B.; Pereira, E. A.

Tabela 3. Resultados das análises físico-químicas dos doces de caqui cv Fuyu adicionados de pinhão.

\begin{tabular}{cccc} 
Parâmetros & \multicolumn{3}{c}{ Amostra } \\
\cline { 2 - 4 } SS ( ${ }^{\circ}$ Brix) & A & B & C \\
Aw & $69,63^{\mathrm{a}} \pm 0,47$ & $70,52^{\mathrm{a}} \pm 0,47$ & $71,00^{\mathrm{a}} \pm 0,00$ \\
Umidade (\%) & $0,776^{\mathrm{a}} \pm 0,002$ & $0,778^{\mathrm{a}} \pm 0,001$ & $0,771^{\mathrm{a}} \pm 0,007$ \\
Cinzas (\%) & $28,58^{\mathrm{a}} \pm 0,08$ & $27,43^{\mathrm{b}} \pm 0,37$ & $25,47^{\mathrm{b}} \pm 0,40$ \\
PH & $0,29^{\mathrm{b}} \pm 0,00$ & $0,30^{\mathrm{b}} \pm 0,01$ & $0,32^{\mathrm{a}} \pm 0,00$ \\
ATT (\%) & $3,65^{\mathrm{c}} \pm 0,01$ & $3,68^{\mathrm{b}} \pm 0,01$ & $3,70^{\mathrm{a}} \pm 0,01$ \\
\hline A: & $0,82^{\mathrm{a}} \pm 0,01$ & $0,80^{\mathrm{ab}} \pm 0,01$ & $0,79^{\mathrm{b}} \pm 0,01$ \\
\hline
\end{tabular}

A: doce de caqui sem pinhão; B: doce de caqui com $5 \%$ de pinhão; C: doce de caqui com 10\% de pinhão; SS: sólidos solúveis; Aw: atividade de água; ATT: acidez total titulável. Médias seguidas da mesma letra minúscula nas linhas não diferem estatisticamente pelo teste de Tukey a 0,05 de significância.

a consequente redução da Aw, melhorando a aparência, o sabor e o rendimento. A padronização deste parâmetro é importante no controle de qualidade.

A Aw determina o limite de água disponível para o crescimento microbiano. De acordo com Franco e Landgraf (2005), os valores limítrofes de atividade de água para multiplicação de bactérias halofílicas, bolores xerofílicos e leveduras osmofílicas são, respectivamente, $0,75,0,65$ e 0,61. Os valores variaram de 0,771 a 0,778, não apresentando diferenças estatísticas; estes resultados demonstram que a adição de pinhão não interfere na Aw. Martins et al. (2007) observaram Aw menor para doces com maior conteúdo de sólidos totais ao analisarem doce em massa de umbu verde e maduro, com variações de 0,76 a 0,86 .

Os teores de umidade variaram entre 25,47 e 28,58\%, e apresentaram diferenças estatísticas devido à adição da semente de pinhão, com resultados próximos aos encontrados por Martins et al. (2011), que avaliaram a estabilidade de doce em massa de banana prata, cujos teores observados apresentaram umidade máxima de 34\%. É possível observar que a umidade variou de acordo com a quantidade de caqui: quanto maior a quantidade da polpa da fruta presente na formulação, maior o teor de umidade. Este comportamento também foi evidenciado por Oliveira et al. (2014), na elaboração de geleias convencionais de umbu-cajá sem o controle da temperatura, durante o armazenamento. Diversamente, Peçanha et al. (2006) encontraram valores mais baixos para goiabadas comerciais do tipo cascão (14,92 a 24,47\%).

Com relação aos resultados de cinzas, a adição de pinhão na composição promoveu um aumento no teor do resíduo mineral fixo de 0,29 a 0,32\%, devido ao elevado teor de minerais da semente de araucária (SILVA et al., 2013). Estes teores estão próximos ao estudo de Freire et al. (2009), que obtiveram valores entre 0,29 e 0,33 na formulação de doce cremoso de goiaba.

Segundo Rosa et al. (2011), a geleificação acontece mais facilmente na faixa de valores de $\mathrm{pH}$ de 3,0 até 3,5. Com valores abaixo de 3,0, ocorre tendência de perda de água da estrutura do gel (MORO et al., 2013). Dentre os requisitos apresentados, destaca-se o $\mathrm{pH}$, que deve estar abaixo de 4,5 (BRASIL, 2005); as amostras analisadas encontram-se entre 3,58 e 3,70. Observe-se que géis mais fortes seriam formados com a diminuição do pH do sistema. Há resultados próximos a 3,79, determinados por Almeida et al. (2009), em goiabada, e abaixo do valor médio de 4,25, encontrado em bananadas comerciais (GODOY et al., 2009).

Os valores de ATT apresentaram diferenças estatísticas e variaram de 0,79 a 0,82\%, teores tais que possibilitaram uma geleificação adequada. Visto que o fruto de caqui é pouco ácido, o uso do ácido cítrico foi o responsável pela elevação no nível de acidez das amostras no processamento. As porcentagens estão próximas ao estudo de Querido et al. (2013), em geleia de caqui, em que os resultados variaram entre 0,78 e 0,81\%, e acima dos valores de 0,26 a 0,68, determinados por Ventura (2004), em doces em massa de valor calórico reduzido de acerola, goiaba vermelha e yacon, desidratados osmoticamente.

\subsection{Análise de cor}

Os resultados da caracterização colorimétrica dos doces cremosos estão apresentados na Tabela 4.

A luminosidade indica o quão claro ou escuro é o doce. Os resultados variaram de 24,46 a 24,84, de forma que a adição de pinhão não foi o fator determinante nessa resposta. O parâmetro está associado à quantidade de sólidos solúveis presentes no produto, como descrito por Moro et al. (2013), em que produtos com teor reduzido de sólidos solúveis apresentam tendência à coloração intensa (clara). De acordo com Wicklund et al. (2005) e Albuquerque (1997), durante a cocção, há formação de produtos derivados de reações enzimáticas e não enzimáticas (reação de Maillard, oxidação de vitaminas, caramelização), as quais são influenciadas pela fruta e por condições de processamento e formulação. Os resultados são intermediários aos doces de pêssego elaborados por Feijó (2013), com valores de $L^{*}$ entre 21,40 e 27,86, e inferiores aos apresentados no estudo de doce de goiaba, 
Elaboração e caracterização de doce cremoso de caqui com adição de sementes da araucária

Bolzan, A. B.; Pereira, E. A.

Tabela 4. Resultados das análises de cor dos doces de caqui cv Fuyu e das formulações adicionados de pinhão.

\begin{tabular}{|c|c|c|c|}
\hline \multirow{2}{*}{ Parâmetros } & \multicolumn{3}{|c|}{ Amostra } \\
\hline & $\mathbf{A}$ & B & C \\
\hline$L^{*}$ & $24,46^{b} \pm 0,07$ & $24,84^{a} \pm 0,05$ & $24,49^{b} \pm 0,08$ \\
\hline$a^{*}$ & $1,11^{b} \pm 0,03$ & $1,78^{a} \pm 0,08$ & $1,79^{a} \pm 0,04$ \\
\hline$b^{*}$ & $6,12^{c} \pm 0,09$ & $7,01^{a} \pm 0,04$ & $6,74^{b} \pm 0,02$ \\
\hline$C^{*}$ & $6,22^{c} \pm 0,09$ & $7,23^{a} \pm 0,02$ & $6,98^{b} \pm 0,02$ \\
\hline$h^{*}$ & $79,68^{a} \pm 0,37$ & $75,77^{b} \pm 0,68$ & $75,11^{b} \pm 0,34$ \\
\hline$\Delta \mathrm{L}^{*}$ & - & $0,38^{a} \pm 0,12$ & $0,13^{a} \pm 0,08$ \\
\hline$\Delta \mathrm{a}^{*}$ & - & $0,66^{a} \pm 0,10$ & $0,68^{a} \pm 0,06$ \\
\hline$\Delta \mathrm{b}^{*}$ & - & $0,89^{a} \pm 0,13$ & $0,62^{a} \pm 0,10$ \\
\hline$\Delta \mathrm{E}_{\mathrm{ab}}{ }^{*}$ & - & $1,18^{a} \pm 0,08$ & $0,94^{b} \pm 0,04$ \\
\hline
\end{tabular}

$L^{*}$ : Luminosidade; $a^{*}:(+)$ vermelho e (-) verde; $b^{*}:(+)$ amarelo e (-) azul; $C^{*}$ : saturação; $h^{*}$ : tonalidade; diferenças absolutas nas coordenadas de cor entre a amostra e o padrão: valores de delta $\left(\Delta \mathrm{L}^{*}, \Delta \mathrm{a}^{*}\right.$ e $\left.\Delta \mathrm{b}^{*}\right) ; \Delta \mathrm{E}^{*}$ : valor total da diferença de cor; A: doce de caqui sem pinhão; B: doce de caqui com $5 \%$ de pinhão; C: doce de caqui com 10\% de pinhão. Médias seguidas da mesma letra minúscula nas linhas não diferem estatisticamente pelo teste de Tukey a 0,05 de significância.

por Menezes et al. (2009), que variaram de 27,55 a 35,94. Estas, portanto, eram amostras mais claras.

Quanto à intensidade de $a^{*}$ (valores positivos representam o vermelho), os dados variaram de 1,11 a 1,79, indicando que a adição de pinhão alterou levemente esta coordenada cromática. Na intensidade de b* (valores positivos representam o amarelo), os resultados estão entre 6,12 e 7,01 , sofrendo alteração com a presença do pinhão. Esses resultados refletiram a cor amarela avermelhada, sendo uma característica deste fruto. Zaghdoudi et al. (2016) fizeram estudos com frutos de caqui, determinando teores de carotenoides considerados como importantes fontes deste pigmento. As pequenas diferenças observadas na cor dos produtos se devem à matéria-prima (cor do fruto e das sementes de pinhão cozidas). Em estudo realizado por Richetti et al. (2014), verificou-se que a ausência de uma cor característica da farinha de pinhão elaborada favorece o uso como ingrediente, pois o mesmo não irá contribuir para uma coloração indesejável do produto. Freire et al. (2009) elaboraram doce cremoso de goiaba e obtiveram valores dos parâmetros $a^{*}$ e $b^{*}$ de cor instrumental de 24,29 a 24,17 e 29,19 a 24,29, respectivamente, ou seja, ocorreram maiores intensidades de amarelo.

O Croma $\left(C^{*}\right)$, que representa a intensidade da cor, mostrou diferença estatística com valores maiores quando foi adicionada a semente de pinhão, significando pequeno aumento na pureza da cor do produto, enquanto que o ângulo $\left(h^{*}\right)$ diminuiu, indicando cor um pouco mais avermelhada que o padrão.

Os valores de delta associados à escala de cor $\left(\Delta \mathrm{L}^{*} \Delta \mathrm{a}^{*} \Delta \mathrm{b}^{*}\right)$ não apresentaram diferenças estatísticas e, portanto, podem ser utilizados como bom indicativo para controle de qualidade e ajustamento de formulações através de tolerâncias estabelecidas entre o padrão e a amostra (MORITRIZ, 2011). Considerando-se o $\Delta \mathrm{E}=3,7$ (PORTERO, 2010) como sendo o valor de acuidade visual, os resultados indicam que as diferenças entre as amostras, mesmo com variações estatisticamente significativas, não podem ser percebidas pela visão humana. Silva (2015), trabalhando com preparado de fruta com massa base de casca de maracujá e aplicação em iogurte, concluiu não haver diferença de cor entre os produtos formulados. Stokes, Fairchild e Berns (1992), citado por Mitterer-Daltoé et al. (2012), consideraram valores de tolerância para $\Delta \mathrm{E}=2,15$; neste caso, as diferenças entre as formulações não são percebidas visualmente. Sob perspectiva industrial, $\Delta \mathrm{E}^{\star}$ com valores de 1,1 até 2,8 unidades na escala CIELAB apresentam-se como um rigoroso padrão de qualidade; valores entre 2,8 a 5,6, como um padrão normal, e verifica-se um baixo padrão, quando os valores se encontram acima de 5,6 (MARTíNEZ et al., 2001).

\subsection{Análise de textura}

As propriedades de textura são importantes componentes na percepção e na aceitabilidade da qualidade de alimento, sendo um reflexo da composição química do alimento e de sua estrutura (DIAS et al., 2011). A diferença entre os valores de parâmetros textura é devida a diferentes mecanismos de formação do gel (KOPJAR et al., 2009). Na Tabela 5, são apresentados os resultados dos perfis de texturas dos doces analisados.

Os atributos de textura são usados para monitorar e controlar a qualidade do produto e a aceitabilidade (CHEN; OPARA, 2013). De modo geral, estes parâmetros estão relacionados aos teores de fruta, umidade, ATT, $\mathrm{pH}$, SS e pectina.

As amostras que continham pinhão apresentaram dureza superior em relação à amostra padrão. A variação observada está relacionada principalmente à presença de amido, como explica Thys et al. (2013), na caracterização e avaliação do amido nativo de pinhão. Observe-se que esta é uma semente fonte de fibra dietética (CORDENUNSI et al., 2004). 
Elaboração e caracterização de doce cremoso de caqui com adição de sementes da araucária

Bolzan, A. B.; Pereira, E. A.

A amostra padrão teve resultados similares para a adesividade aos obtidos por Dias et al. (2011), em geleias processadas com a casca de banana cv Prata. Houve um aumento na adesividade das amostras B e C, ou seja, a presença do amido do pinhão promoveu efeito, verificado por meio de um aumento significativo neste parâmetro. O aumento da energia de remoção da sonda durante o teste de textura das amostras B e C pode estar relacionado à variação do teor de SS, resultado similar ao estudo de Godoy et al. (2009).

A elasticidade da estrutura é afetada pela acidez, enrijecendo as fibras da rede devido à hidrólise da pectina (SANTOS et al., 2012). Os valores de elasticidade não diferiram entre si e estão de acordo com Almeida et al. (2009), em goiabadas desenvolvidas com edulcorantes $(0,954$ a 0,988$)$.

Segundo Besbes et al. (2009), a coesividade representa o quão bem o alimento resiste a uma segunda deformação, em relação à primeira; os valores encontrados na agregação de valor da geleia de tâmara variaram de 0,51 a 0,77 . Não houve diferença estatística entre as amostras, revelando que o pinhão não interferiu neste parâmetro.

A gomosidade é um parâmetro secundário, associado a firmeza e coesividade (BOURNE, 2002), e sua variação é reflexo destas. Os resultados apontaram maiores valores para as amostras B e C, como consequência do aumento da dureza, promovida possivelmente pela gelatinização do amido e pela força simulada para romper as ligações internas do gel (KALVIAINEN et al., 2000). Os resultados encontram-se abaixo dos determinados por Souza et al. (2015), em geleia elaborada com amora cv Brazos.

A Análise de Componentes Principais (ACP) foi utilizada para elucidar as relações encontradas entre as variações das formulações e entender como estas podem afetar as características dos produtos desenvolvidos (Figura 1a, b).

Tabela 5. Resultados das análises do perfil de textura dos doces de caqui cv Fuyu adicionados de pinhão.

\begin{tabular}{lrrr} 
& \multicolumn{3}{c}{ Amostra } \\
\cline { 2 - 4 } \multicolumn{1}{c}{ Parâmetro } & A & B & C \\
Dureza (g) & $74,37^{\mathrm{c}} \pm 1,36$ & $106,57^{\mathrm{a}} \pm 0,12$ & $98,10^{\mathrm{b}} \pm 1,39$ \\
Adesividade (-gs) & $39,57^{\mathrm{b}} \pm 1,22$ & $76,60^{\mathrm{a}} \pm 2,16$ & $75,73^{\mathrm{a}} \pm 3,72$ \\
Elasticidade & $0,96^{\mathrm{a}} \pm 0,01$ & $0,96^{\mathrm{a}} \pm 0,01$ & $0,95^{\mathrm{a}} \pm 0,01$ \\
Coesividade & $0,41^{\mathrm{a}} \pm 0,01$ & $0,44^{\mathrm{a}} \pm 0,00$ & $0,45^{\mathrm{a}} \pm 0,01$ \\
Gomosidade & $28,99^{\mathrm{b}} \pm 0,36$ & $47,33^{\mathrm{a}} \pm 0,61$ & $44,65^{\mathrm{a}} \pm 0,70$ \\
\hline
\end{tabular}

A: doce de caqui sem pinhão; B: doce de caqui com $5 \%$ de pinhão; C: doce de caqui com 10\% de pinhão. Médias seguidas da mesma letra minúscula nas linhas não diferem estatisticamente pelo teste de Tukey a 0,05 de significância.

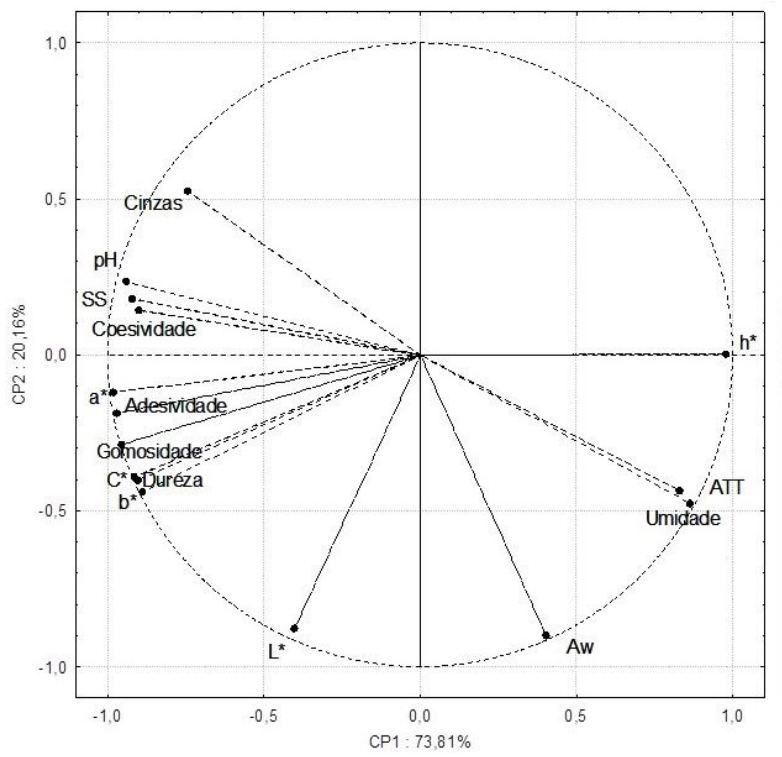

(a)

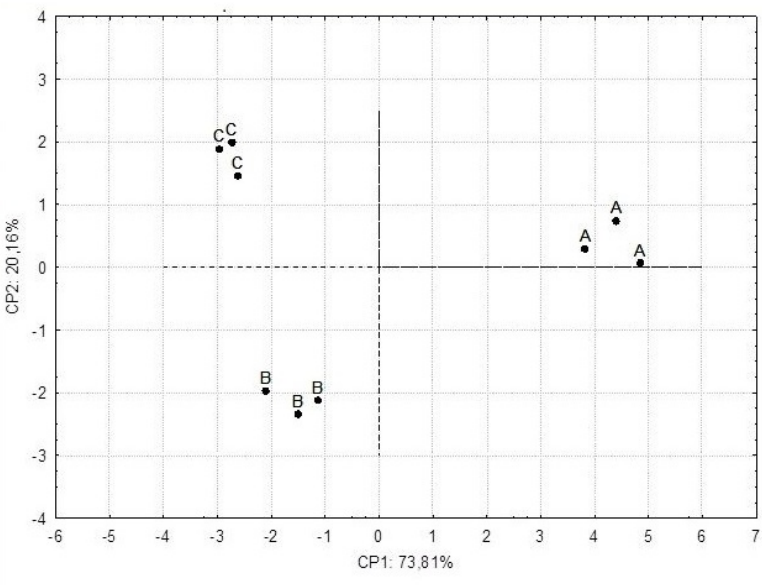

(b)

Figura 1. Projeção espacial da ordenação dos vetores das variáveis físicas e físico-químicas (a) e posição das amostras (b) nas duas componentes principais. A: doce de caqui sem pinhão; B: doce de caqui com 5\% de pinhão; C: doce de caqui com 10\% de pinhão; SS: sólidos solúveis; Aw: atividade de água; ATT: acidez total titulável; L*: Luminosidade; a*: (+) vermelho e (-) verde; b*: (+) amarelo e (-) azul; C*: saturação; $h^{*}$ : tonalidade. 
Elaboração e caracterização de doce cremoso de caqui com adição de sementes da araucária

Bolzan, A. B.; Pereira, E. A.

Por meio do método estatístico de múltiplas variáveis as análises físicas e físico-químicas com maior correlação embasaram o julgamento e a importância das componentes mais elevadas na explicação da variância dos produtos desenvolvidos, denotando assim, a homogeneidade em cada grupo e explicando $93,97 \%$ da variabilidade dos dados, sendo que a CP1 e CP2, retêm $73,81 \%$ e $20,16 \%$, respectivamente. O primeiro descritor representado pela tonalidade $\left(h^{*}\right)$ separa o doce sem adição de pinhão, amostra A (padrão), diferindo-a das demais formulações e comandando o eixo positivo da CP1. O eixo da CP2 é altamente influenciado pelos vetores negativos cujas variáveis que mais contribuíram para formação do segundo grupo distinto foram os parâmetros $+a^{*}$ (teor de vermelho), $+b^{*}$ (teor de amarelo) e $C^{*}$ (intensidade ou pureza da cor), além das características similares de textura representadas pela dureza, adesividade e gomosidade da amostra B; enquanto que o terceiro grupo da CP2, formado pelos valores positivos de SS, pH, cinzas e as medidas de coesividade, apresentam os maiores índices e promovem a clara separação das amostras de doce C. A variável elasticidade apresentou comunalidade baixa e foi removida do conjunto de dados.

\section{Conclusão}

As formulações analisadas estão de acordo com os padrões físico-químicos do produto. O incremento da farinha de semente de pinhão, devido às características neutras no espaço de cor $L^{*} a^{*} b^{*}$, não promoveu modificações perceptíveis ao olho humano, na diferença total das coordenadas absolutas da cor. As propriedades mecânicas associadas com a textura do doce foram alteradas favoravelmente, com aumento da dureza, adesividade e gomosidade. As amostras apresentaram ausência dos micro-organismos pesquisados, sendo consideradas seguras sob o ponto de vista microbiológico e reafirmando, ainda, que foram respeitadas as boas práticas de fabricação. Concluiu-se, neste estudo, que é viável a elaboração de doce cremoso de caqui cv Fuyu, com a substituição parcial da polpa pela adição de diferentes concentrações de sementes de pinhão. O produto é inovador, o que pode ajudar a promover e aumentar o interesse pela preservação do bioma, gerando renda e aporte nutricional.

\section{Referências}

AKUTSU, R. C.; BOtELHO, R. A.; CAMARGO, E. B.; SÁVIO, K. E. O.; ARAÚJO, W. C. Adequação das boas práticas de fabricação em serviços de alimentação. Revista de Nutrição, v. 18, n. 3, p. 419-427, 2005. http://dx.doi.org/10.1590/S141552732005000300013

ALBUQUERQUE, J. P. Fatores que influenciam no processamento de geleias e geleiadas de frutas. Ciência e Tecnologia de Alimentos, v. 31, n. 1, p. 1-8, 1997.
ALMEIDA, E. L.; RAMOS, A. M.; BINOTI, M. L.; CHAUCA, M. C.; STRINGHETA, P. C. Análise de perfil de textura e aceitabilidade sensorial de goiabadas desenvolvidas com diferentes edulcorantes. Revista Ceres, v. 56, n. 6, p. 697-704, 2009.

BESBES, S.; DRIRA, L.; BLECKER, C.; DEROANNE, C.; ATTIA, H. Adding value to hard date (Phoenix dactylifera L.): compositional, functional and sensory characteristics of date jam. Food Chemistry, v. 112, n. 2, p. 406-411, 2009. http:// dx.doi.org/10.1016/j.foodchem.2008.05.093.

BOLZAN, A. B.; PEREIRA, E. A. Análise sensorial de geleia de caqui cv Fuyu adicionada de sementes de pinhão cozido. In: SIMPÓSIO DE TECNOLOGIA QUÍMICA, 3., 2016, Pato Branco. Anais... Pato Branco: Universidade Tecnológica Federal do Paraná, 2016. p. 1-6.

BOURNE, M. C. Food texture and viscosity: concept and measurement. 2nd ed. New York: Elsevier Science and Food Technology, 2002.

BRASIL. Agência Nacional da Vigilância Sanitária. Resolução CNNPA n 12, de 1978. Normas Técnicas Especiais, do Estado de São Paulo, relativas a alimentos (e bebidas), para efeito em todo território brasileiro. Diário Oficial [da] República Federativa do Brasil, Brasília, DF, 24 ago. 1978.

BRASIL. Agência Nacional da Vigilância Sanitária. Resolução RDC n 12, de 2 de janeiro de 2001. Estabelece os padrões microbiológicos sanitários para alimentos. Diário Oficial [da] República Federativa do Brasil, Brasília, DF, 2 jan. 2001a.

BRASIL. Ministério do Meio Ambiente. Resolução CONAMA n 278, de 24 de maio de 2001. Dispõe contra corte e exploração de espécies ameaçadas de extinção da flora da Mata Atlântica. Diário Oficial [da] República Federativa do Brasil, Brasília, DF, 18 junho. 2001b. Seção 1, p. 51-52.

BRASIL. Ministério da Saúde. Agência Nacional da Vigilância Sanitária. Resolução RDC n² 272, de 22 de setembro de 2005. Regulamento técnico para produtos de vegetais, produtos de frutas e cogumelos comestíveis. Diário Oficial [da] República Federativa do Brasil, Brasília, DF, 23 set. 2005.

CAETANO, P. K.; DAIUTO, É. R.; VIEITES, R. L. Característica físico-química e sensorial de geleia elaborada com polpa e suco de acerola. Brazilian Journal of Food Technology, v. 15, n. 3, p. 191-197, 2012. http://dx.doi.org/10.1590/S198167232012005000011.

CAMARGO FILHO, W. P.; MAZZEI, A. R.; ALVES, H. S. Mercado de caqui: variedades, estacionalidade e preços. Informações Econômicas, v. 33, n. 10, p. 81-87, 2003.

CHEN, L.; OPARA, U. L. Texture measurement approaches in fresh and processed foods. Food Research International, v. 51, n. 2 , p. 823-835, 2013. http://dx.doi.org/10.1016/j.foodres.2013.01.046.

CITADINI-ZANETTE, V.; PEREIRA, J. L.; JARENKOW, J. A.; KLEIN, A. S.; SANTOS, R. Estrutura da sinúsia herbácea em Floresta Ombrófila Mista no Parque Nacional de Aparados da 
Elaboração e caracterização de doce cremoso de caqui com adição de sementes da araucária Bolzan, A. B.; Pereira, E. A.

Serra, sul do Brasil. Revista Brasileira de Biociências, v. 9 , n. 1, p. 56-63, 2011.

COMPANHIA NACIONAL DE ABASTECIMENTO - CONAB. Pinhão (semente). Brasília, 2014. 21 p. Conjuntura Especial. Disponível em: <http://www.conab.gov.br/OlalaCMS/uploads/ arquivos/14_09_09_12_36_06_pinhaosementesetembro_2014. pdf>. Acesso em: 3 jul. 2015.

CORDENUNSI, B. R.; MENEZES, E. W.; GENOVESE, M. I.; DOLLI, C.; SOUZA, A. G.; LAJOLO, F. M. Chemical composition and glycemic index of Brazilian pine (Araucaria angustifolia) seeds. Journal of Agricultural and Food Chemistry, v. 52, n. 11, p. 3412-3416, 2004. PMid:15161207. http://dx.doi.org/10.1021/ jf034814l.

DIAS, C. S.; BORGES, S. V.; QUEIROZ, F.; PEREIRA, P. A. P. Influência da temperatura sobre as alterações físicas, físicoquímicas e químicas de geleia da casca de banana (Musa spp.) Cv. Prata durante o armazenamento. Revista do Instituto Adolfo Lutz, v. 70, n. 1, p. 28-34, 2011.

ELIAS, N. F.; BERBERT, P. A.; MOLINA, M. A. B.; VIANA, A. P.; DIONELLO, R. G.; QUEIROZ, V. A. V. Avaliação nutricional e sensorial de caqui cv Fuyu submetido à desidratação osmótica e secagem por convecção. Ciência e Tecnologia de Alimentos, v. 28, n. 2, p. 322-328, 2008. http://dx.doi.org/10.1590/S010120612008000200009

FEIJÓ, L. Caracterização físico-química e sensorial de doces de pêssego com diferentes teores de açúcar. 2013. 34 f. Dissertação (Mestrado em Qualidade e Segurança Alimentar)Escola Superior Agrária de Bragança, Bragança, 2013.

FIGUEIREDO FILHO, A.; ORELLANA, E.; NASCIMENTO, F.; DIAS, A. N.; INOUE, M. T. Produção de sementes de Araucaria angustifolia em plantio e em floresta natural no Centro-Sul do estado do Paraná. Floresta, v. 41, n. 1, p. 155-162, 2011. http:// dx.doi.org/10.5380/rf.v41i1.21196.

FRANCO, B. D. G. M.; LANDGRAF, M. Microbiologia dos alimentos. São Paulo: Atheneu, 2005. 182 p.

FREIRE, M. T. A.; PETRUS, R. R.; HASHIDA, J. C.; FAVAROTRINDADE, C. S. Avaliação física, química e sensorial de doce cremoso de goiaba acondicionado em bisnaga plástica. Brazilian Journal of food technology, v. 12, n. 3, p. 172-180, 2009.

GODOY, R. C. B.; MATOS, E. L. S.; SANTOS, D. V.; AMORIM, T. S.; WASZCZYNSKYJ, N.; SOUSA NETO, M. A. Estudo da composição físico-química e aceitação de bananadas comerciais por meio de análise multivariada. Revista do Instituto Adolfo Lutz, v. 68, n. 3, p. 373-380, 2009.

INSTITUTO ADOLFO LUTZ - IAL. Métodos físico-químicos para análise de alimentos. 4. ed. São Paulo: Instituto Adolfo Lutz, 2008. 1020 p.

INSTITUTO AGRONÔMICO DO PARANÁ - IAPAR. Previsão climática: agrometeorologia. Curitiba, 2015. Disponível em: <http:// www.iapar.br/modules/conteudo/conteudo.php?conteudo=2084> Acesso em: 3 set. 2016.

KALVIAINEN, N.; ROININEN, K.; TUORILA, H. Sensory characterization of texture and flavor of high viscosity gels made with different thickeners. Journal of Texture Studies, v. 31, n. 4, p. 407-420, 2000. http://dx.doi.org/10.1111/j.1745-4603.2000.tb00299.x.

KATO, T.; RIBEIRO, K. P.; BORDONAL, V. C.; SILVA, M. B. S.; OLIVEIRA, A. F.; SEIBEL, N. F. Avaliação da qualidade de doces de frutas agroindustriais do Norte do Paraná. Revista Brasileira de Produtos Agroindustriais, v. 15, n. 2, p. 173-182, 2013. http://dx.doi.org/10.15871/1517-8595/rbpa.v15n2p173-182.

KOPJAR, M.; PILIŽOTA, V.; TIBAN, N. N.; ŠUBARIĆ, D.; BABIĆ, J.; AČKAR, Đ.; SAJDL, M. Strawberry jams: influence of different pectins on colour and textural properties. Czech Journal of Food Sciences, v. 27, n. 1, p. 20-28, 2009.

LEITE JÚNIOR, B. R. C.; OLIVEIRA, P. M.; CASTRO, R. L. E.; LAMAS, J. M. N.; MARTINS, E. M. F. Desenvolvimento e caracterização de doce de goiaba cremoso adicionado de farinha de okara. Segurança Alimentar e Nutricional, v. 20, n. 1, p. 111-121, 2013.

MARTINELI, M.; ALVES, A. A. R.; FIGUEIREDO, G. M.; REZENDE, C. M.; FONSECA, M. J. O. Caqui cv. 'Mikado': análise de compostos voláteis em frutos adstringentes e destanizados. Ciência Rural, v. 43, n. 8, p. 1516-1521, 2013. http://dx.doi. org/10.1590/S0103-84782013000800028.

MARTíneZ, J. A.; MELGOSA, M.; PÉREZ, M. M.; HITA, E.; NEGUERUELA, A. I. Visual and instrumental color evaluation in red wines. Food Science and Technology, v. 7, n. 5, p. 439-445, 2001. http://dx.doi.org/10.1106/VFAT-5REN-1WK2-5JGQ.

MARTINS, G. A. S.; FERRUA, F. Q.; MESQUITA, K. S.; BORGES, S. V.; CARNEIRO, J. D. S. Estabilidade de doces em massa de banana prata. Revista do Instituto Adolfo Lutz, v. 70, n. 3, p. 332-340, 2011

MARTINS, M. L. A.; BORGES, S. V.; DELIZA, R.; CASTRO, F. T.; CAVALVANTE, N. B. Características de doce em massa de umbu verde e maduro e aceitação. Pesquisa Agropecuária Brasileira, v. 42, n. 9, p. 1329-1333, 2007. http://dx.doi.org/10.1590/S0100$204 \times 2007000900015$.

MENEZES, C. C.; BORGES, S. V.; CIRILLO, M. A.; FERRUA, F. Q.; OLIVEIRA, L. F.; MESQUITA, K. S. Caracterização física e físicoquímica de diferentes formulações de doce de goiaba (Psidium guajava L.) da cultivar Pedro Sato. Ciência e Tecnologia de Alimentos, v. 29, n. 3, p. 618-625, 2009. http://dx.doi.org/10.1590/ S0101-20612009000300025.

MINAS GERAIS. Governo do Estado. Secretaria de Estado de Agricultura, Pecuária e Abastecimento de Minas Gerais. Subsecretaria do Agronegócio. Caqui. Belo Horizonte, 2015. Disponível em: <http://agricultura.mg.gov.br/images/documentos/ perfil_caqui_dez_2015[1].pdf>. Acesso em: 5 jan. 2016. 
Elaboração e caracterização de doce cremoso de caqui com adição de sementes da araucária Bolzan, A. B.; Pereira, E. A.

MIQUELIM, J. N. Avaliação reológica e físico-químicas de bombons recheados com preparado de morango, laranja e maracujá em base açúcar fondant, gordura hidrogenada e chocolate branco. 2006. 91 f. Dissertação (Mestrado em Tecnologia Bioquímico-Farmacêutica)- Departamento de Tecnologia de Alimentos, Universidade de São Paulo, São Paulo, 2006.

MITTERER-DALTOÉ, M. L.; PETRY, F. C.; WILLE, D. F.; TREPTOW, R. O.; MARTINS, V. M. V.; QUEIROZ, M. I. Chemical and sensory characteristics of meat from Nellore and Crioulo Lageano breeds. International Journal of Food Science and Technology, v. 47, n. 10, p. 2092-2100, 2012. http://dx.doi.org/10.1111/j.13652621.2012.03075.x.

MORITRIZ, A. R. Existe cor em nossas vidas: a colorimetria aplicada em nossos dias. São Paulo: Ed. BRASEQ, 2011. 176 p.

MORO, G. M. B.; RODRIGUES, R. S.; COSTA, J. A. V.; PIZATO, S.; MACHADO, W. R. C. Avaliação da rotulagem e qualidade físicoquímica de geleias de uva comercializadas na cidade do Rio Grande-RS. Revista Brasileira de Tecnologia Agroindustrial, v. 7, n. 1, p. 897-910, 2013.

NEIRO, E. S.; NANNI, M. R.; ROMAGNOLI, F.; CAMPOS, R. M.; CEZAR, E.; CHICATI, M. L.; OLIVEIRA, R. B. Análise de cor de para discriminação de seis variedades de cana-de-açúcar em quatro épocas de colheita no ano. In: SIMPÓSIO BRASILEIRO DE SENSORIAMENTO REMOTO - SBSR, 16., 2013, Foz do Iguaçu. Anais... São José dos Campos: Instituto Brasileiro de Pesquisas Espaciais, 2013. p. 274-281.

NUNES, G. D. G.; TOMÉ, P. H. F.; FRAGIORGE, E. J. Elaboração e análise sensorial do vinho de caqui (diospyros kaki L.) cv. Fuyu. In: JORNADA CIENTÍFICA DA FAZU, 8., 2009, Uberada. Anais... Uberaba: Faculdades Associadas de Uberaba, 2009. p. 44-49.

OLIVEIRA, E. N. A.; SANTOS, D. C.; ROCHA, A. P. T.; GOMES, J. P.; SILVA, W. P. Estabilidade de geleias convencionais de umbu-cajá durante o armazenamento em condições ambientais. Revista Brasileira de Engenharia Agrícola e Ambiental, v. 18, n. 3, p. 329-337, 2014. http://dx.doi.org/10.1590/S141543662014000300013

PARANÁ. Governo do Paraná. Secretaria de Estado do Meio Ambiente e Recursos Hídricos. Instituto Ambiental do Paraná. Portaria IAP $n^{\circ}$ 048, de 22 de março de 2011. Institui os procedimentos para controle da exploração do PINHÃO e define outras providencias. Diário Oficial do Estado do Paraná, Curitiba, PR, 22 mar. 2011.

PEÇANHA, D. A.; NEVES, T. G.; VERRUMA-BERNARDI, M. R.; DELIZA, R.; ARAÚJO, K. G. L.; KAJISHIMA, S.; PINHEIRO, M. S. Qualidade microbiológica, físico-química e sensorial de goiabada tipo cascão produzida na região Norte do Estado do Rio de Janeiro. Brazilian Journal of Food Technology, v. 9 , n. 1, p. 25-32, 2006.

PORTERO, P. P. Avaliação instrumental da correspondência de cor de resinas compostas em função de escala de referência, tonalidade, composição e espessura. 2010. 138 f. Tese (Doutorado em Dentística Restauradora)-Programa de Pós-graduação em Ciências Odontológicas, Universidade Estadual Paulista, Araraquara, 2010.

QUERIDO, A. F.; SILVA, C. S. A.; GUIMARÃES, D. H. P.; ALVES, G. L. Persimmon pulp and jelly: chemical characterization and rheological behavior. Brazilian Journal of Applied Technology for Agricultural Science, v. 6, n. 2, p. 97-103, 2013.

RICHETTI, A. E.; PEDROTTI, V.; HUBER, E.; BIASI, V. Elaboração e caracterização físico-química de farinha da semente de pinhão (Araucaria Angustifólia). In: MOSTRA NACIONAL DE INICIAÇÃO CENTíFICA E TECNOLOGIA INTERDICIPLICAR, 7., 2014, Araquari. Anais... Araquari: Instituto Federal Catarinense, 2014. p. 1-5.

ROSA, N. C.; TRINTIM, L. T.; CORRÊA, R. C. G.; VIEIRA, A. M. S.; BERGAMASCO, R. Elaboração de geleia de abacaxi com hortelã zero açúcar: processamento, parâmetros físico-químicos e análise sensorial. Revista Tecnológica, p. 83-89, 2011. Edição Especial V Simpósio de Engenharia, Ciência e Tecnologia de Alimentos.

SANTOS, K. M. A.; GONÇALVES, C. A. S.; PIRES, V. C. F.; FLORÊNCIO, I. M.; QUEIROZ, M. S. R. Avaliação sensorial de geleia de acerola com pimenta. In: ENCONTRO NACIONAL DE EDUCAÇÃO, CIÊNCIA E TECNOLOGIA, 2012, Campina Grande. Anais... Campina Grande: Universidade Estadual da Paraíba, 2012. p. 1-10.

SCHVEITZER, B.; ROSA, A. M.; GRANEMANN, P.; KLOCK, A. L. S.; RIZZATTI, I. M.; FOPPA, T. Caracterização química de pinhões - sementes de araucária angustifólia - em diferentes formas de preparo. Revista Interdisciplinar de Estudos em Saúde, v. 3, n. 1, p. 93-104, 2014.

SENTER, S. D.; CHAPMAN, G. W.; FORBUS JUNIOR, W. R.; PAYNE, J. A. Sugar and nonvolatile acid composition of persimmons during maturation. Journal of Food Science, v. 56, n. 4, p. 989-991, 1991. http://dx.doi.org/10.1111/j.1365-2621.1991.tb14623.x.

SILVA, C. J. Desenvolvimento de preparo de frutas com massa base de casca de maracujá e aplicação em iogurte. 2015, 76 f. Dissertação (Mestrado em Engenharia de Processos Químicos e Bioquímicos)-Escola de Engenharia Mauá, Instituto Mauá de Tecnologia, São Caetano do Sul, 2015.

SILVA, C. M.; ZANQUI, A. B.; SOUZA, A. H. P.; GOHARA, A. K.; VISENTAINER, J. V.; MATSUSHITA, M. Caracterização química e quantificação dos minerais da semente de araucaria angustifolia (BERTOL.) O. Kuntze. In: ENCONTRO INTERNACIONAL DE PRODUÇÃO CIENTíFICA CESUMAR, 8., 2013, Maringá. Anais... Maringá: Editora CESUMAR, 2013. p. 1-4.

SOUZA, A. V.; RODRIGUES, R. J.; GOMES, E. P.; GOMES, G. P.; VIEITES, R. L. Caracterização bromatológica de frutos e geleias de amora-preta. Revista Brasileira de Fruticultura, v. 37, n. 1, p. 13-19, 2015. http://dx.doi.org/10.1590/0100-2945-037/14.

SOUZA, T. C. Alimentos: propriedades físico-químicas. 2. ed. Rio de Janeiro: Cultura Médica, 2001. 240 p. 
Elaboração e caracterização de doce cremoso de caqui com adição de sementes da araucária

Bolzan, A. B.; Pereira, E. A.

THYS, R. C. S.; AIRES, A. G.; MARCZAK, L. D. F.; NOREÑA, C. P. Z. The effect of acid hydrolysis on the technological functional properties of pinhão (Araucaria brasiliensis). Ciência e Tecnologia de Alimentos, v. 33, n. 1, p. 89-94, 2013. http:// dx.doi.org/10.1590/S0101-20612013000500014.

VENDRUSCOLO, C. T.; MOREIRA, A. S.; VENDRUSCOLO, J. L. $S$. Tecnologia de frutas e hortaliças: geleias, doces cremosos e em massa. Pelotas: Editora Universitária UFPel, 2009.

VENTURA, F. C. Desenvolvimento de doce de fruta em massa funcional de valor calórico reduzido, pela combinação de goiaba vermelha e yacon desidratados osmoticamente e acerola. 2004. 194 f. Dissertação (Mestrado em Tecnologia de
Alimentos)-Faculdade de Engenharia de Alimentos, Universidade Estadual de Campinas, Campinas, 2004

WICKLUND, T.; ROSENFELD, H. J.; MARTISEN, B. K.; SUNDFOR, M. W.; LEA, P.; BRUNN, T.; BLOMHOFF, R.; HAFFNER, K. Antioxidant capacity and colour of strawberry jam as influenced by cultivar and storage conditions. Food Science and Technology, v. 38, n. 4, p. 380-391, 2005.

ZAGHDOUDI, K.; FRAMBOISIER, X.; FROCHOT, C.; VANDERESSE, R.; BARTH, D.; KALTHOUM-CHERIF, J.; BLANCHARD, F.; GUIAVARC'H, Y. Response surface methodology applied to Supercritical Fluid Extraction (SFE) of carotenoids from Persimmon (Diospyros kaki L.). Food Chemistry, v. 208, p. 209-219, 2016. PMid:27132842. http://dx.doi.org/10.1016/j.foodchem.2016.03.104. 